\title{
MTA1 promotes metastasis of MPM via suppression of E-cadherin
}

\author{
Caihua Xu, Fei Hua, Yihuan Chen, Haoyue Huang, Wenxue Ye, Yunsheng Yu and Zhenya Shen ${ }^{*}$
}

\begin{abstract}
Background: Metastasis-associated gene 1(MTA1) has been identified as an oncogene in many tumors, and aberrant MTA1 expression has been linked to carcinogenesis and metastasis. We aim to investigate the mechanism of MTA1 and metastasis in malignant pleural mesothelioma (MPM).

Methods: Real-time polymerase chain reaction (PCR) and immunohistochemical staining were employed to detect MTA1 and E-cadherin expression in MPM tissues and corresponding adjacent tissues. Stable clone with knock-down of MTA1 was generated with shRNA via lentivirus technology in MPM cell lines. Wound-healing assay, transwell assay and PCR array were carried out for detecting invasion and migration of MPM cells. Luciferase reporter assay was performed to validate the effect of MTA1 on E-cadherin.
\end{abstract}

Results: MTA1 expression is up-regulated in MPM and shown a negative correlation with E-cadherin expression. MTA1 could enhance the invasion and migration of MPM cells via suppressing the expression of E-cadherin. MTA1 overexpression is associated with pathology, metastasis and survival rate of MPM patients.

Conclusions: MTA1 plays an important role in Epithelial-to-mesenchymal transition (EMT) to promote metastasis via suppressing E-cadherin expression, resulting in a poor prognosis in MPM. MTA1 is a novel biomarker and indicative of a poor prognosis in MPM patients.

Keywords: MTA1, E-cadherin, Malignant pleural mesothelioma, Metastasis

\section{Background}

Malignant pleural mesothelioma (MPM) is considered as one of the highest aggressive tumor arising from the cells lining serosal cavities, mostly resulting from the occupational exposure to asbestos fibers [1]. Although great efforts have been made toward improving diagnosis and treatment [2], there are no efficacious therapies for MPM patients presently, and therefore the overall survival is extremely poor. Thus, the investigation of developing the novel therapeutics, especially molecular targeting therapy, is very important for the patients with MPM.

MTA (metastasis-associated gene) is a newly discovered family of cancer progression-related genes and their encoded products. MTA are integral parts of nucleosome remodeling and histone deacetylation (NuRD) complexes, function as transcriptional co-repressors which regulate varieties pathways, including hormonal action, epithelial-

\footnotetext{
* Correspondence: uuzyshen@126.com

Department of Cardiovascular Surgery of the First Affiliated Hospital and Institute for Cardiovascular Science, Soochow University, Suzhou 215000, China
} 
They were included between December 2008 and November 2013 at the First Affiliated Hospital of Soochow University (Suzhou, China) and the First Affiliated Hospital of Nanjing Medical University (Nanjing, China). The patients were followed up for a median period of 12 months (range, 3-28 months) after operation and their complete clinical data were collected. The correct diagnosis was assessed by an experienced pathologist and the staging of MPM by a clinical oncologist according to AJCC/UICC Guidelines version 7.2010 MPM. Adjacent tissue was located within $3 \mathrm{~cm}$ of the edge of the tumor tissue (Additional file 1: Fig. 1S). The study was approved by the Ethical Committee of the First Affiliated Hospital of Soochow University (Suzhou, China) and the First Affiliated Hospital of Nanjing Medical University (Nanjing, China), and written informed consent was obtained from the each patient.

\section{DNA and RNA preparation}

Total RNA was extracted from fresh frozen tissue specimens using TRIzol method (Invitrogen, Shanghai, China) and RNA quality was detected by NanoDrop 2000 and A260/A280 was between 1.95 and 2.05. cDNA was synthesized using reverse transcriptase kit (TAKARA, Tokyo, Japan) according to the manufacturers' protocol.

\section{Real-time PCR analysis}

MTA1 and E-cadherin mRNA levels were measured by real-time PCR using SYBR Premix Ex Taq (TAKARA, Tokyo, Japan). MTA1 and E-cadherin transcription values were normalized against the expression of $\beta$ actin. Amplification conditions, primers, and probes sequences for MTA1 and $\beta$-actin were from the work by Zhu X et al. [9] and for E-cadherin were the same as those in the work by Martínez-Estrada et al. [11]. All procedures are in agreement with MIQE guidelines.

\section{Cell culture}

MSTO-211H, NCI-H2452 and 293 T cell lines (ATCC, Manassas, VA) were employed for the present study. MSTO-211H and H2452 were origin from the patients with mesothelioma and cultured in RPMI 1640 medium supplemented with $10 \%$ fetal bovine serum (Invitrogen, Carlsbad, CA), while 293 T were origin from human embryonic kidney cells and cultured in DMEM high glucose medium supplemented with $10 \%$ fetal bovine serum. All cells were maintained in a humidified $37{ }^{\circ} \mathrm{C}$ incubator with $5 \% \mathrm{CO}_{2}$.

\section{Lentivirus production and transduction}

To generate plasmid-expressing MTA1-shRNA, doublestranded oligonucleotides were cloned into pLL3.7 vector (gifted by D. Yun Chen, Nanjing Medical University, China) and named pLL3.7-shMTA1. The sequences of
MTA1-shRNA used are ccggtGACCACCGACAGATAC GTG ttcaagaga CACGTATCTGTCGGTGGTCTTTTT Tg. The uppercase letters represent MTA1-specific sequence, and lowercase letters represent hairpin sequences. Recombinant lentivirus was generated from 293 T cells using calcium phosphate precipitation. MSTO- $211 \mathrm{H}$ and H2452 were transfected with lentivirus using polybrene $(8 \mu \mathrm{g} / \mathrm{ml})$.

\section{Western-blotting assay}

Proteins were extracted from cultured cells, quantitated using a protein assay (bicinchoninic acid [BCA] method; Beyotime, Shanghai, China). Proteins were fractionated by sodium dodecyl sulfate polyacrylamide gel electrophoresis, transferred to polyvinylidene fluoride (PVDF) membrane, blocked in $4 \%$ dry milk at room temperature for 1 hour, and immunostained with primary antibodies at $4{ }^{\circ} \mathrm{C}$ over-night using anti-MTA1 (1:2000, Abcam, Cambridge, MA), anti-E-cadherin (1:1000; Abcam, Cambridge, MA), and anti-GAPDH (1:1000, Kangchen,China). The results were visualized via a chemiluminescent detection system (Pierce ECL Substrate Western blot detection system; Thermo, Rockford, IL) and exposed in Molecular Imager ChemiDoc XRS System (Bio-Rad, Hercules, CA).

\section{Cell proliferation assay}

Cells were seeded into 96 -well plates $\left(6.0 \times 10^{3}\right.$ cells per well). Cell viability was assessed by cell-counting kit- 8 assay (CCK-8, Beyotime, Shanghai, China). The absorbance of each well was read on a spectrophotometer (Thermo) at $450 \mathrm{~nm}$ (A450). Five independent experiments were performed in quintuplicate.

\section{Wound-healing assay}

Cells were seeded in six-well plates and cultured to confluence. Wounds of $2-\mathrm{mm}$ width were created with a plastic scriber and the floating cells were washed away thrice with phosphate buffered saline (PBS). After incubation in a serum-free medium for 48 hours, cultures were observed and photos were taken under a microscope. A minimum of five randomly chosen areas was measured.

\section{Transwell invasion assay}

The invasive ability of the cells was investigated using Transwells (8- $\mu \mathrm{m}$ pore size; Corning Costar Corp, Bedford, MA) put into the 24-well plates. First, $50 \mu \mathrm{l}$ Matrigel ( $50 \mu \mathrm{g} / \mathrm{ml}$; BD Biosciences, San Jose, CA) was added onto each surface of the chamber, incubated for 2 hours for solidification, then the supernatant was washed away with warm PBS. MSTO-211H and $\mathrm{H} 2452$ were suspended in RPMI 1640 containing $2 \%$ fetal bovine serum. A total of $100 \mu \mathrm{l}$ of the cell suspension $\left(5 \times 10^{4}\right.$ cells $)$ was added to the upper chamber coated with Matrigel, and $400 \mu \mathrm{l}$ of 
RPMI 1640 containing $10 \%$ fetal bovine serum was added to the lower compartment. After incubation for 48 hours at $37{ }^{\circ} \mathrm{C}$ in a $5 \% \mathrm{CO}_{2}$ humidified incubator, the Matrigel and cells on the upper surface of the filter were removed with cotton swabs and the cells that invaded into the lower surface were fixed with $2 \%$ paraformaldehyde, stained with crystal violet. Then the filters were removed from the chambers, air-dried on the precleaned slides and applied with cover-slides using resina. Images were taken under an inverted microscope (Olympus Corp, Tokyo, Japan) at $\times 100$ magnifications over three random fields in each well. ImageJ $1.45 \mathrm{~s}$ software (National Insititutes of Health, Bethesda, MD) was used for integrated optical density analysis. Each experiment was performed in triplicate.

\section{RT-PCR array}

Total RNA was extracted from MSTO-211H, MSTO211H-shMTA1, H2452 and H2452-shMTA and reverse transcribed to cDNA. Subsequently, cDNA was amplified by PCR using 23 Super Array PCR master mix (SuperArray Bioscience, Frederick, MD) and then RT-PCR was carried out using the Human Tumor Metastasis RT2 Profiler PCR array (SuperArray Bioscience, Frederick, MD) in an ABI PRISM7900 system (Applied Biosystems, Foster City, CA), according to the manufacturer's instructions.

\section{Luciferase report assay}

The promoter of E-cadherin was synthesized artificially and cloned into the luciferase reporter vector (Promega, Madison, WI). MSTO-211H and H2452 cells were seeded into 24-well plates and cotransfected with MTA1-siRNA (pooled siGENOME SMART pool MTA1 siRNA [50 nM per well]; Dhamacon, Chicago, IL), and luciferase reporter vectors using Lipofectamine 2000 (Invitrogen, Carlsbad, CA), following the instructions. Zero, 12 and 24 hours after incubation, cells were collected and firefly and Renilla luciferase activity was measured with the dualluciferase reporter assay system (Promega). All results were gained through three independent experiments.

\section{TGF- $\beta 1$ Inducing EMT of MPM cells}

The MPM cells were cultured in vitro and induced by transforming growth fact beta1 (TGF- $\beta 1,5 \mathrm{ng} / \mathrm{ml}$ ) for $0 \mathrm{~h}, 24 \mathrm{~h}, 48 \mathrm{~h}$. The morphological characteristics of cells were observed by microscope (Olympus Corp, Tokyo, Japan) and the expression of MTA1 was detected by real-time PCR.

\section{Immunohistochemical staining}

Tissues were fixed in $4 \%$ paraformaldehyde and cut from paraffin block to $5 \mu \mathrm{m}$ thickness. After dewaxing with xylene and rehydration with a graded series of ethanol, the slides were heated in the autoclave for three minutes using citrate buffer ( $\mathrm{PH}$ 6.0) and incubated with primary antibody MTA1 (1:1000, Abcam, Cambridge, MA), E-cadherin (1:1000; Abcam, Cambridge, MA) at $4{ }^{\circ} \mathrm{C}$ overnight. Blocking serum or antibody dilution buffer was prepared as Negative controls. The primary antibodies utilized were all the same as for Western blot analysis. Photographs were taken by microscope (Nikon, ECLIPSE 50i) and software NIS-Elements v4.0. Average values of integrated optical density (IOD) were obtained from five random fields per slide by using Image-Pro Plus software (v5.0). Every data was detected three times at least.

\section{Statistical analysis}

Statistical analysis was performed using GraphPad Prism (version 5.01; GraphPad Software, Inc, La Jolla, CA) statistical software. The Student's $t$ test and paired $t$ test were used to analyze significance between independent groups and paired materials, respectively. The correlation test was used to analyze the correlation between MTA1 and E-cadherin. The $X^{2}$ test was used to test the significance of observed differences in proportions except when the cells size was less than 5 (Fisher's exact tests). The significance was accepted as p value was less than 0.05 .

\section{Results}

MTA1 expression is up-regulated in MPM and shown a negative correlation with E-cadherin expression

We investigated 65 pairs of MPM and corresponding adjacent specimens using real-time PCR and immunohistochemistry. MTA1 RNA level in MPM was significantly higher compared to adjacent tissues $(p<0.0001$; Fig.1a). However, E-cadherin RNA level was just contrary $(p<0.0001$; Fig.1b). Moreover, a negative correlation was observed between MTA1 and E-cadherin expression in MPM samples $(r=-0.6250 ; p<0.0001$; Fig. 1c). To further evaluate the correlation between MTA1 and E-cadherin expression, two cases with different MTA1 expression levels are shown in Fig. 1d: CASE 1 (strong positive) and CASE 2 (weak positive). The level of E-cadherin was down-regulated in CASE 1 compared to CASE 2. Additionally, statistical analysis of IOD values stained with E-cadherin in MTA1-high group and MTA1-low group are shown in the histogram in Fig. $1 \mathrm{e}$ and $\mathrm{f}(p<0.0001)$. The results suggested that MTA1 expression was up-regulated and negatively correlated to E-cadherin expression in MPM.

\section{MTA1 could enhance the invasion and migration of MPM cells in vitro}

In vitro, both two MPM cell lines (MSTO-211H and H2452) were transduced by pLL3.7-shMTA1 and detected the expression of MTA1 by real-time PCR and Western-blot (Fig. 2a, b, and c). These results indicated 


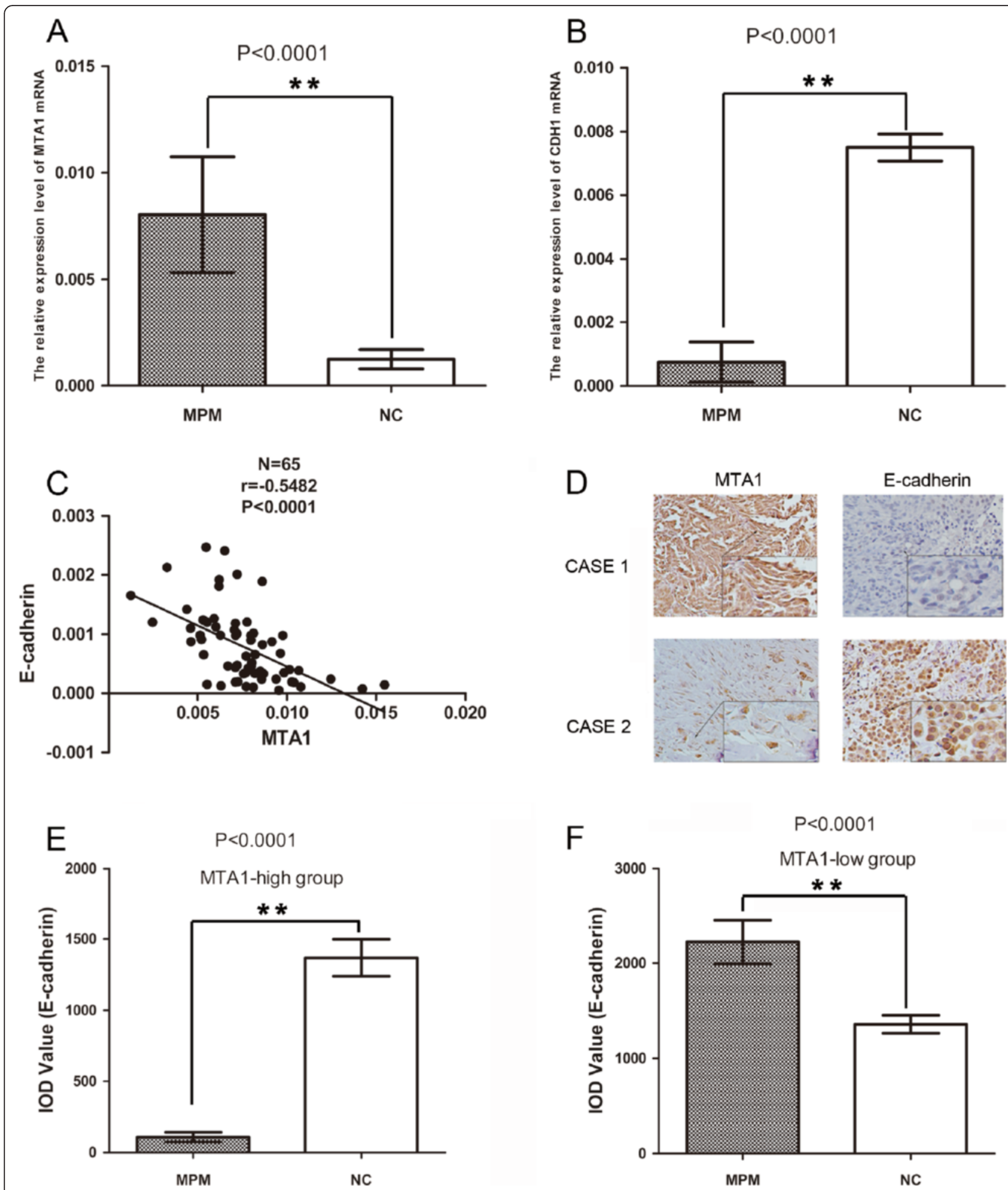

Fig. 1 Expression and correlation of MTA1 and E-cadherin transcripts in MPM patients. a Significantly higher RNA expression of MTA1 in tumor specimens than in the adjacent tissue samples $(p<0.001)$. $\mathbf{b}$ Significantly decreased RNA expression of E-cadherin in tumor specimens in comparison with adjacent tissues $(p<0.001)$. c A negative correlation between MTA1 and E-cadherin in tumor samples $(r=-0.5482, p<0.0001)$. $\mathbf{d}$ Immunohistochemical staining of E-cadherin in MTA1 overexpression tumor tissue (CASE 1) and MTA1 low expression tumor tissue (CASE 2) in vivo. Data are represented as mean $\pm S D$. ${ }^{*} p<0.05,{ }^{* *} p<0.001$. e and $\mathbf{f}$ Average value of integrated optical density (IOD) of E-cadherin in MTA1-high group and MTA1-low group were assessed by analyzing five fields per slide and recorded in the histogram. MTA1, metastasis-associated gene 1; MPM, malignant pleural mesothelioma 

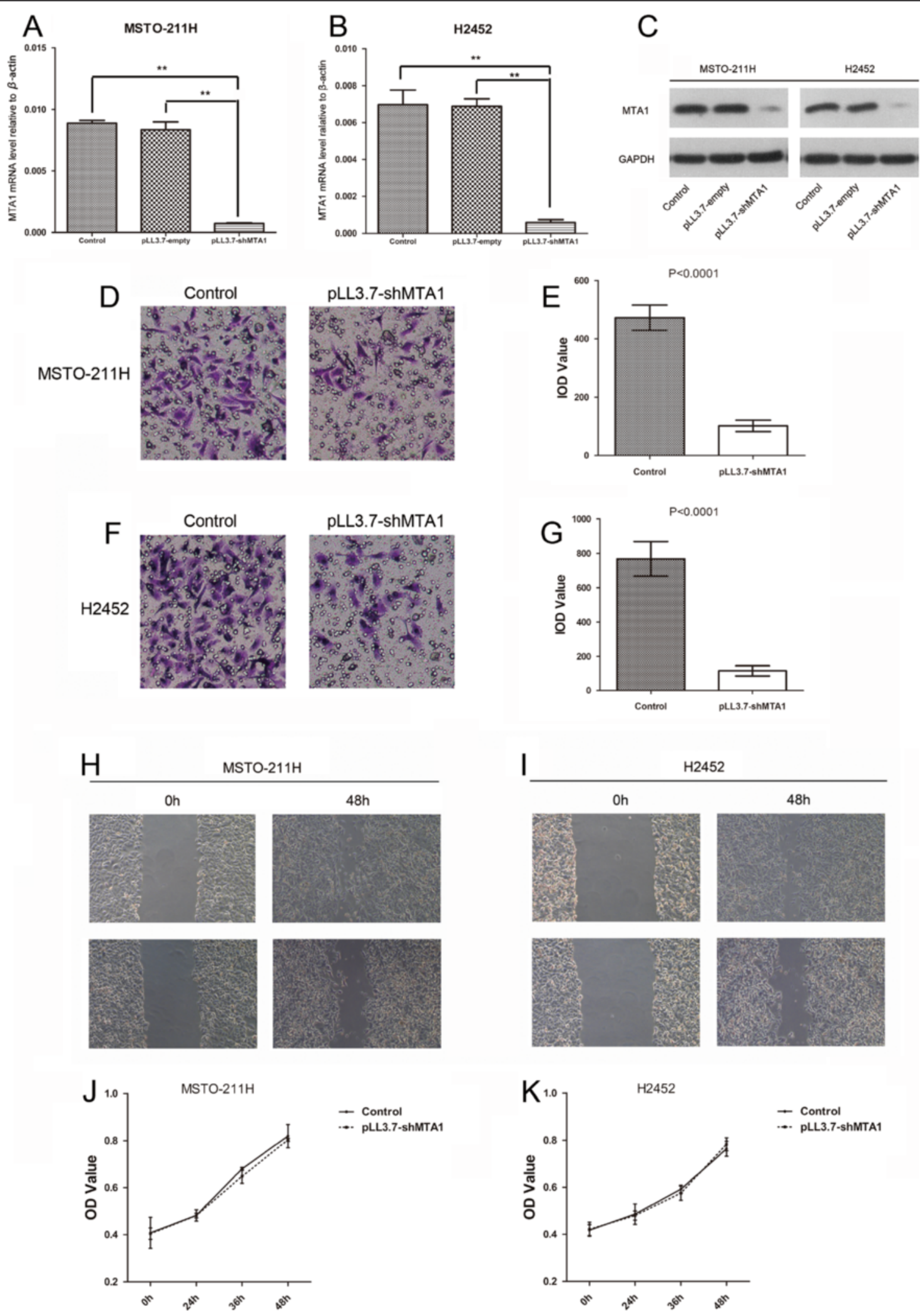

Fig. 2 (See legend on next page.) 


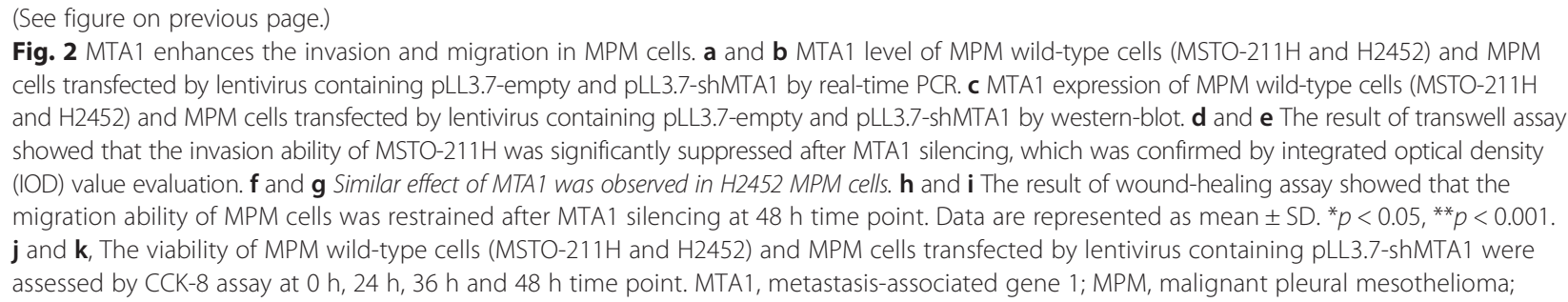

expression of MTA1 was significantly reduced by the lentivirus. As illustrated, the invasion and migration abilities of both MSTO- $211 \mathrm{H}$ and $\mathrm{H} 2452$ were evidently suppressed when MTA1 was down-regulated by pLL3.7shMTA1 (Fig. 2d, f, h and i). And the quantification of the invasion abilities of MSTO-211H and $\mathrm{H} 2452$ are presented in Fig. 2e and g corresponding with Fig. 2d and $\mathrm{f}$. Moreover, We found that MTA1 silencing could not affect cell proliferation (Fig. $2 \mathrm{j}$ and $\mathrm{k}$ ).

\section{MTA1 knockdown induces increase of E-cadherin expression in MPM cells}

A variety of EMT genes were screened out after MTA1 down-regulation via the PCR array and nine genes whose variation larger than two folds were picked. Then we verified the expression of these nine genes in 65 samples by real-time PCR. We found E-cadherin was upregulated most after MTA1 silenced in both MSTO-211H and H2452 (Fig. 3a and b). In order to confirm the negative regulatory effect of MTA1 on E-cadherin, we also detected the E-cadherin protein in both two cells by Western-blot. As shown in Fig. 3c, E-cadherin protein expression was increased significantly in both two cells transduced by pLL3.7-shMTA1. Subsequently, we performed luciferase report assay in MSTO-211H and H2452 transfected with MTA1-siRNA and the luciferase activity of E-cadherin was enhanced significantly with transfection time increases (Fig. 3d and e). Moreover, we found the mRNA expression of MTA1 was up-regulated in TGF- $\beta 1$ stimulated MPM cells (Fig 3f) which was according with the result of Suresh B et al. [12]. Taken together, these findings suggest that the decreased expression of MTA1 could enhance E-cadherin expression in MPM cells.

\section{MTA1 overexpression is associated with a poor prognosis of MPM}

A total of 65 MPM tissues were divided into two groups using a semiquantitative immunoreactivity scoring system (IRS), as reported elsewhere [9, 10, 13, 14]. Concomitant cytoplasmicstaining was not counted. Category A documented the intensity of immunostaining as 0 (no immunostaining), 1(weak immunostaining), 2 (moderate immunostaining), and 3 (strong immunostaining).
Category B documented the percentage of immunoreactive cells as 0 (none), 1 (<10\%), 2(10-50\%), 3 (51-80\%), and 4 (80\%). Multiplication of categories A and B resulted in an IRS ranging from 0 to 12 (negative [IRS, 0] versus weak [IRS, 1-4] versus moderate [IRS, 5-8] versus strong [IRS, 9-12]) for each tumor. Both percent positivity of cells and staining intensity were decidedin a doubleblinded manner. Interobserver and intraobserver variability was negligible. Tumors with moderate or strong expression (IRS 4) were considered to show MTA1 overexpression, whereas tumors with negative or weak expression (IRS < 4) were considered to show MTA1 downexpression. The relationship of MTA1 expression levels and clinicopathological features were shown in Table 1. Significant higher TNM stage $(p<0.01)$, metastasis (lymph node metastasis and distant metastasis, $p<0.01$ ) were observed in high-MTA1 expression group. We also found MTA1 level in sarcomatoid and biphasic malignant mesotheliomas were higher than the level in epithelioid malignant mesothelioma $(p<0.05)$. There was no correlation between MTA1 expression level and sex, age, as shown in Table 1. Additionally, our findings indicated that MPM patients with a high expression level of MTA1 had a significantly shorter overall survival compared with patients with a low expression level of MTA1 and 10 months median survival compared with 19 months median survival after operation $(p<0.01$, Fig. $3 g)$. These results strongly indicate that MTA1 overexpression contribute to the poor prognosis of MPM patients.

\section{Discussion}

Malignant pleural mesothelioma (MPM), one of the most deadly human carcinomas, is an aggressive tumor which originates from the mesothelial cells of serosal tissues [15]. Metastasis is the major cause of treatment failure in patients with MPM and its molecular mechanisms are still not clear and are under intensive investigation. To elucidate the mechanisms of metastasis is essential for improving surgery and treatment outcome, especially for selecting and customizing chemotherapy.

In this study, for the first time, we reported that MTA1 gene and protein in MPM specimens was markedly upregulated compared to adjacent tissues, and MTA1 could 

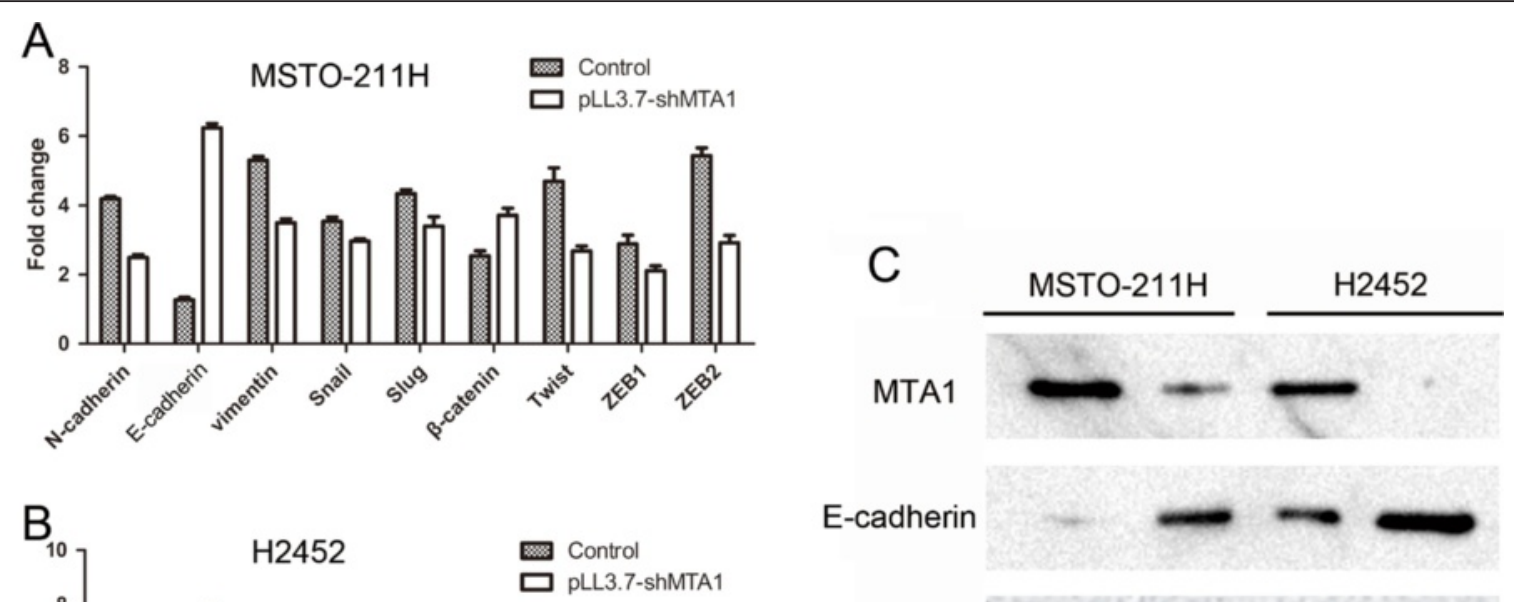

E-cadherin
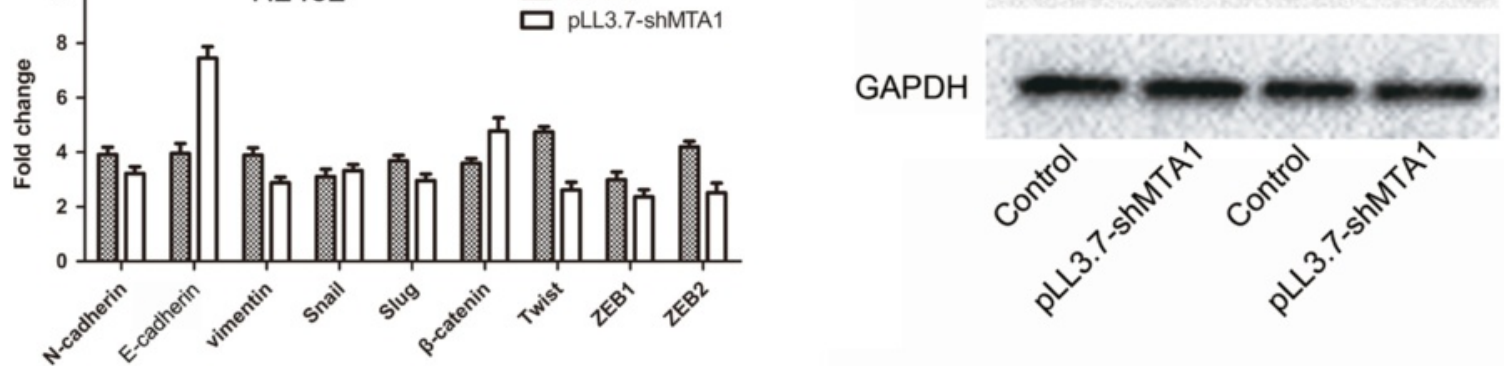

D

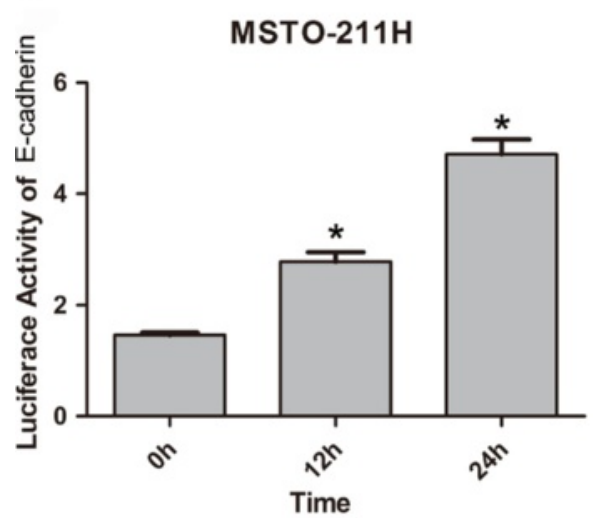

E
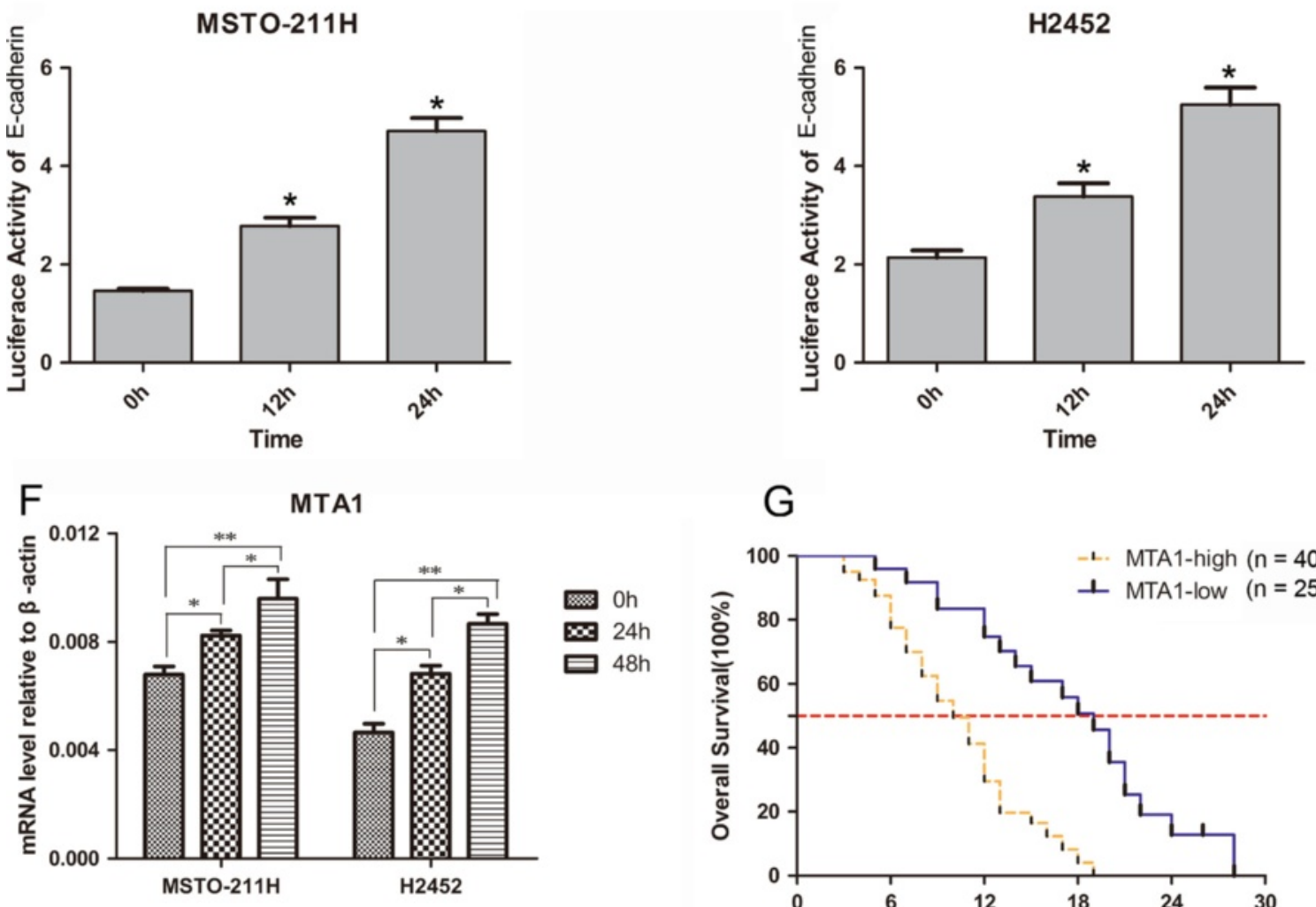

G

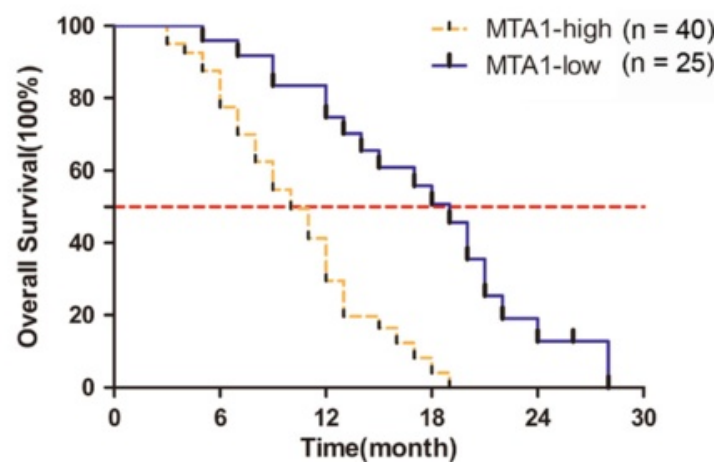

Fig. 3 (See legend on next page.) 
(See figure on previous page.)

Fig. 3 MTA1 suppressed the expression of E-cadherin and was up-regulated by TGF- $\beta 1$ inducing. a and $\mathbf{b}$ Nine metastasis genes varied larger than two folds were picked via PCR array and were verified by real-time PCR in MPM cells. c Western-blot assay validated the result of PCR array of negative trend of MTA1 and E-cadherin in MPM cells. $\mathbf{d}$ and $\mathbf{e}$ Luciferase report assay demonstrated that E-cadherin transcription was gradually enhanced in MPM cells transfected by lentivirus containing pLL3.7-shMTA1 in a time-dependent manner. $\mathbf{f}$ MTA1 mRNA expression was up-regulated in TGF- $\beta 1$-stimulated MPM cells. g MPM patients with a high expression level of MTA1 had a significantly shorter overall survival compared with patients with a low expression level of MTA1. Data are represented as mean \pm SD. ${ }^{*} p<0.05$, ${ }^{* *} p<0.001$. MTA1, metastasis-associated gene 1; MPM, malignant pleural mesothelioma; TGF- $\beta 1$, transforming growth factor beta 1; PCR, polymerase chain reaction

enhance the metastasis of MPM cells by direct regulation of E-cadherin. Additionally, the relationship of MTA1 level and clinicopathological features in Table 1 suggests that MTA1 overexpression was associated with the poor prognosis of MPM patients. With all findings taken together, we hypothesized that MTA1 potentially play an oncogenic role in promoting metastasis of MPM.

MTA1 has been considered to be related with the poor prognosis due to increase the migration and invasion of various tumors $[6,8,16-19]$. As a dual function co-regulator, MTA1 operates as a transcriptional

Table 1 Correlation of MTA1 expression and clinicopathological features of MPM

\begin{tabular}{|c|c|c|c|c|c|}
\hline \multirow[t]{2}{*}{ Characteristics } & \multirow[t]{2}{*}{ All patients } & \multicolumn{2}{|c|}{ MTA1 expression } & \multirow[t]{2}{*}{$P$} & \multirow[b]{2}{*}{$x^{2}$} \\
\hline & & Low & High & & \\
\hline $\mathrm{N}$ & 65 & 25 & 40 & & \\
\hline Sex & & & & 0.455 & 0.559 \\
\hline Male & 35 & 12 & 23 & & \\
\hline Female & 30 & 13 & 17 & & \\
\hline Age (years) & & & & 0.842 & 0.040 \\
\hline$<60$ & 27 & 10 & 17 & & \\
\hline$\geq 60$ & 38 & 15 & 23 & & \\
\hline Histology & & & & 0.038 & 6.524 \\
\hline Epithelioid & 20 & 13 & 7 & & \\
\hline Sarcomatoid & 25 & 7 & 18 & & \\
\hline Biphasic & 20 & 5 & 15 & & \\
\hline TNM stage $^{a}$ & & & & 0.003 & 8.990 \\
\hline$|/| \mid$ & 29 & 17 & 12 & & \\
\hline III/IV & 36 & 8 & 28 & & \\
\hline Lymph node Metastasis & & & & 0.001 & 11.714 \\
\hline Yes & 38 & 8 & 30 & & \\
\hline No & 27 & 17 & 10 & & \\
\hline Distant Metastasis & & & & $<0.001$ & 14.562 \\
\hline Yes & 35 & 6 & 29 & & \\
\hline No & 30 & 19 & 11 & & \\
\hline
\end{tabular}

aTNM stage was based on AJCC/UICC Guidelines version 7.2010 MPM. MTA1, metastasis-associated protein 1; MPM, Malignant pleural mesothelioma repressor of ER- $\alpha, B R C A 1$ and $\mathrm{p} 21$ and as a transcriptional activator of BCAS3 and Pax5 [20-24]. Some studies have indicated that down-regulation of MTA1 by RNAi leads to an increase expression of E-cadherin which acts as a key role in epithelial-to-mesenchymal transition in cancer cells [25-27]. Moreover, Wenhao Weng et al. have elucidated that MTA1, together with Snail or Slug, acted to directly repress the promoter activity of E-cadherin in ESCC (esophageal squamous cell carcinoma) cells very recently [28]. Coincidentally, we verified that MTA1 could enhance the epithelial-tomesenchymal transition by repressing the promoter activity of E-cadherin in MPM cells.

Epithelial-to-mesenchymal transition (EMT) is a cellular process which is considered to be involved in embryo-morphogenesis, fibrosis and tumor metastasis $[29,30]$. EMT could enhance not only the ability of migration and invasion, but also the resistance to apoptosis, senescence and drug resistance in cancer [31]. EMT process always accompany by a reduced expression of E-cadherin, cyto-keratins (CK), and $\beta$ catenin (in the membrane), and an increased expression of Snail, Slug, Twist, ZEB1, ZEB2, N-cadherin, vimentin, a-smooth muscle actin (aSMA), S100A4, and matrix metalloproteinases (MMP) [31-33]. In particular, repressing the gene expression of E-cadherin could notably promote the EMT which is considered as a primary reason of tumor metastasis. According to our study, the poor prognosis of patients with high MTA1expression was correlated with E-cadherin, the very classical metastasis suppressor.

\section{Conclusion}

Our study identified the negative correlation of MTA1 and E-cadherin in 65 MPM cases, both of which were associated with TNM stage, metastasis and histological types and proved the regulatory function of MTA1 on Ecadherin through direct binding of its promoter. However, as a limit of the number of MPM cases and cell types, more elaborate research will be requireda for further exploration of MTA1 in tumorigenesis and metastasis. Thus, MTA1 potentially served as a therapeutic target for the treatment of MPM. 


\section{Additional file}

Additional file 1: Figure 1S. MTA1 protein expression in tumor tissue and adjacent tissue of one MPM patient. A and B, immunohistochemical staining of MTA1 in tumor specimen in comparison with adjacent tissue. (TIF $2636 \mathrm{~kb}$ )

\section{Abbreviation}

MTA: Metastasis-associated gene; MTA1: Metastasis-associated gene 1; MPM: Malignant pleural mesothelioma; PCR: Polymerase chain reaction; EMT: Epithelial-to-mesenchymal transition; NuRD: Nucleosome remodeling and histone deacetylation; CK: Cyto-keratins; aSMA: A-smooth muscle actin MMP: Matrix metalloproteinases.

\section{Competing interests}

The authors declare that they have no competing interests

\section{Authors' contributions}

CX conceived and designed the experiments. CX, FH wrote the article. $Y C$ and $\mathrm{HH}$ prepared the patient samples. $\mathrm{CX}$ and $\mathrm{YC}$ performed the experiments. WY, YY and ZS collected and analyzed the datas. All authors read and approved the final manuscript

\section{Acknowledgment}

The authors thank D. Yun Chen from Nanjing Medical University for providing pLL-3.7 plasmids.

Received: 20 October 2015 Accepted: 9 December 2015 Published online: 21 December 2015

\section{Reference}

1. Tsao AS, Wistuba I, Roth JA, Kindler HL. Malignant pleural mesothelioma. J Clin Oncol. 2009;27:2081-90.

2. Carbonelli C, Rapicetta C, Cesario A. Surgery and survival of patients with diagnosis of malignant pleural mesothelioma. J Thorac Oncol. 2015;10:e92-3.

3. Toh Y, Nicolson GL. The role of the MTA family and their encoded proteins in human cancers: molecular functions and clinical implications. Clin Exp Metastasis. 2009:26:215-27.

4. Manavathi B, Kumar R. Metastasis tumor antigens, an emerging family of multifaceted master coregulators. J Biol Chem. 2007;282:1529-33.

5. Balasenthil S, Broaddus RR, Kumar R. Expression of metastasis-associated protein 1 (MTA1) in benign endometrium and endometrial adenocarcinomas. Hum Pathol. 2006:37:656-61.

6. Kidd M, Modlin IM, Mane SM, Camp RL, Eick G, Latich I. The role of genetic markers-NAP1L1, MAGE-D2, and MTA1-in defining small-intestinal carcinoid neoplasia. Ann Surg Oncol. 2006;13:253-62.

7. Giannini R, Cavallini A. Expression analysis of a subset of coregulators and three nuclear receptors in human colorectal carcinoma. Anticancer Res. 2005:25:4287-92.

8. Hamatsu T, Rikimaru T, Yamashita Y, Aishima S, Tanaka S, Shirabe K, et al. The role of MTA1 gene expression in human hepatocellular carcinoma. Oncol Rep. 2003;10:599-604.

9. Zhu X, Guo Y, Li X, Ding Y, Chen L. Metastasis-associated protein 1 nuclear expression is associated with tumor progression and clinical outcome in patients with non-small cell lung cancer. J Thorac Oncol. 2010;5:1159-66.

10. Li $Y$, Chao $Y$, Fang $Y$, Wang J, Wang $M$, Zhang $H$, et al. MTA1 promotes the invasion and migration of non-small cell lung cancer cells by downregulating miR-125b. J Exp Clin Cancer Res. 2013:32:33.

11. Martinez-Estrada OM, Lettice LA, Essafi A, Guadix JA, Slight J, Velecela V, et al. Wt1 is required for cardiovascular progenitor cell formation through transcriptional control of Snail and E-cadherin. Nat Genet. 2010;42:89-93.

12. Pakala SB, Singh K, Reddy SD, Ohshiro K, Li DQ, Mishra L, et al. TGF-beta1 signaling targets metastasis-associated protein 1, a new effector in epithelial cells. Oncogene. 2011;30:2230-41

13. Deng L, Yang H, Tang J, Lin Z, Yin A, Gao Y, et al. Inhibition of MTA1 by ERalpha contributes to protection hepatocellular carcinoma from tumor proliferation and metastasis. J Exp Clin Cancer Res. 2015;34:128.

14. Song $Q$, Zhang $H$, Wang $M$, Song $W$, Ying M, Fang $Y$, et al. MTA1 promotes nasopharyngeal carcinoma growth in vitro and in vivo. J Exp Clin Cancer Res. 2013;32:54.
15. Kumar P, Kratzke RA. Molecular prognostic markers in malignant mesothelioma. Lung Cancer. 2005:49 Suppl 1:S53-60.

16. Toh $Y$, Ohga T, Endo K, Adachi E, Kusumoto H, Haraguchi M, et al. Expression of the metastasis-associated MTA1 protein and its relationship to deacetylation of the histone $\mathrm{H} 4$ in esophageal squamous cell carcinomas. Int J Cancer. 2004;110:362-7.

17. Hofer MD, Kuefer R, Varambally S, Li H, Ma J, Shapiro Gl, et al. The role of metastasis-associated protein 1 in prostate cancer progression. Cancer Res. 2004:64:825-9.

18. Mahoney MG, Simpson A, Jost M, Noe M, Kari C, Pepe D, et al. Metastasisassociated protein (MTA)1 enhances migration, invasion, and anchorageindependent survival of immortalized human keratinocytes. Oncogene. 2002:21:2161-70.

19. Hofer MD, Menke A, Genze F, Gierschik P, Giehl K. Expression of MTA1 promotes motility and invasiveness of PANC-1 pancreatic carcinoma cells. Br J Cancer. 2004;90:455-62.

20. Mazumdar A, Wang RA, Mishra SK, Adam L, Bagheri-Yarmand R, Mandal M, et al. Transcriptional repression of oestrogen receptor by metastasisassociated protein 1 corepressor. Nat Cell Biol. 2001;3:30-7.

21. Molli PR, Singh RR, Lee SW, Kumar R. MTA1-mediated transcriptional repression of BRCA1 tumor suppressor gene. Oncogene. 2008;27:1971-80.

22. Li DQ, Pakala SB, Reddy SD, Ohshiro K, Peng SH, Lian Y, et al. Revelation of p53-independent function of MTA1 in DNA damage response via modulation of the p21 WAF1-proliferating cell nuclear antigen pathway. J Biol Chem. 2010;285:10044-52.

23. Gururaj AE, Singh RR, Rayala SK, Holm C, den Hollander $P$, Zhang $H$, et al. MTA1, a transcriptional activator of breast cancer amplified sequence 3 . Proc Natl Acad Sci U S A. 2006;103:6670-5.

24. Balasenthil S, Gururaj AE, Talukder AH, Bagheri-Yarmand R, Arrington T, Haas BJ, et al. Identification of Pax5 as a target of MTA1 in B-cell lymphomas. Cancer Res. 2007;67:7132-8.

25. Jiang $\mathrm{Q}$, Zhang $\mathrm{H}$, Zhang P. ShRNA-mediated gene silencing of MTA influenced on protein expression of ER alpha, MMP-9, CyclinD1 and invasiveness, proliferation in breast cancer cell lines MDA-MB-231 and MCF-7 in vitro. J Exp Clin Cancer Res. 2011:30:60.

26. Rao $Y$, Wang $H$, Fan $L$, Chen $G$. Silencing MTA1 by RNAi reverses adhesion, migration and invasiveness of cervical cancer cells ( $\mathrm{SiHa}$ ) via altered expression of p53, and E-cadherin/beta-catenin complex. J Huazhong Univ Sci Technolog Med Sci. 2011:31:1-9.

27. Zhang KJ, Wang DS, Zhang SY, Jiao XL, Li CW, Wang XS, et al. The E-cadherin repressor slug and progression of human extrahepatic hilar cholangiocarcinoma. J Exp Clin Cancer Res. 2010;29:88.

28. Weng W, Yin J, Zhang Y, Qiu J, Wang X. Metastasis-associated protein 1 promotes tumor invasion by downregulation of E-cadherin. Int J Oncol. 2014;44:812-8

29. Acloque $H$, Adams MS, Fishwick K, Bronner-Fraser M, Nieto MA. Epithelial-mesenchymal transitions: the importance of changing cel state in development and disease. J Clin Invest. 2009:119:1438-49.

30. Thiery JP, Acloque H, Huang RY, Nieto MA. Epithelial-mesenchymal transitions in development and disease. Cell. 2009;139:871-90.

31. Voulgari A, Pintzas A. Epithelial-mesenchymal transition in cancer metastasis: mechanisms, markers and strategies to overcome drug resistance in the clinic. Biochim Biophys Acta. 2009:1796:75-90

32. Mani SA, Guo W, Liao MJ, Eaton EN, Ayyanan A, Zhou AY, et al. The epithelial-mesenchymal transition generates cells with properties of stem cells. Cell. 2008;133:704-15

33. Zeisberg M, Neilson EG. Biomarkers for epithelial-mesenchymal transitions. J Clin Invest. 2009;119:1429-37. 